\title{
Analytical Solution of Two-Dimensional Sine-Gordon Equation
}

\author{
Alemayehu Tamirie Deresse $\left(\mathbb{D}\right.$, Yesuf Obsie Mussa ${ }^{(D)}$, and Ademe Kebede Gizaw 1
}

Department of Mathematics, College of Natural Sciences, Jimma University, Ethiopia

Correspondence should be addressed to Yesuf Obsie Mussa; yesufobsie@gmail.com

Received 26 December 2020; Revised 8 March 2021; Accepted 9 April 2021; Published 3 May 2021

Academic Editor: Maria L. Gandarias

Copyright (c) 2021 Alemayehu Tamirie Deresse et al. This is an open access article distributed under the Creative Commons Attribution License, which permits unrestricted use, distribution, and reproduction in any medium, provided the original work is properly cited.

\begin{abstract}
In this paper, the reduced differential transform method (RDTM) is successfully implemented for solving two-dimensional nonlinear sine-Gordon equations subject to appropriate initial conditions. Some lemmas which help us to solve the governing problem using the proposed method are proved. This scheme has the advantage of generating an analytical approximate solution or exact solution in a convergent power series form with conveniently determinable components. The method considers the use of the appropriate initial conditions and finds the solution without any discretization, transformation, or restrictive assumptions. The accuracy and efficiency of the proposed method are demonstrated by four of our test problems, and solution behavior of the test problems is presented using tables and graphs. Further, the numerical results are found to be in a good agreement with the exact solutions and the numerical solutions that are available in literature. We have showed the convergence of the proposed method. Also, the obtained results reveal that the introduced method is promising for solving other types of nonlinear partial differential equations (NLPDEs) in the fields of science and engineering.
\end{abstract}

\section{Introduction}

Nonlinear phenomena, which appear in many areas of scientific fields such as solid-state physics, plasma physics, fluid dynamics, mathematical biology, and chemical kinetics, can be modeled by partial differential equations. A broad class of analytical and numerical solution methods were used to handle these problems. Recently, several research on the physical phenomena of the diverse fields of engineering and science was carried out, see for example [1-9] and the references therein.

The nonlinear sine-Gordon equation (SGE), a type of hyperbolic partial differential equation, is often used to describe and simulate the physical phenomena in a variety of fields of engineering and science, such as nonlinear waves, propagation of fluxions, and dislocation of metals, for details see [10] and the references therein. Because the sine-Gordon equation has many kinds of soliton solutions, it has attracted wide spread attention [11]. The sine-Gordon equation was first discovered in the nineteenth century in the course of study of various problems of differential geometry [12]. In the early 1970s, it was first realized that the sine-Gordon equation led to kink and antikink (so-called solitons) [13]. As one of the crucial equations in nonlinear science, the sine-Gordon equation has been constantly investigated and solved numerically and analytically in recent years [10, 1418]. Different scholars employed different methods to solve the one-dimensional sine-Gordon equation, for example, the Adomian decomposition method (ADM) [19-23], the EXP function method [24], the homotopy perturbation method (HPM) [25-27], the homotopy analysis method (HAM) [28], the variable separated ODE method [29, 30], and the variational iteration method (VIM) [31,32]. Further, Shukla et al. [33] obtained numerical solution of the twodimensional nonlinear sine-Gordon equation using a localized method of approximate particular solutions. Baccouch [34] developed and analyzed an energy-conserving local discontinuous Galerkin method for the two-dimensional SGE on Cartesian grids. Duan et al. [35] proposed a numerical model based on the lattice Boltzmann method to obtain the numerical solutions of the two-dimensional generalized sine-Gordon equation, and the method was 
extended to solve the nonlinear hyperbolic telegraph equation as indicated in [36].

The main aim of this study is to obtain the approximate analytical solutions for the two-dimensional nonlinear sineGordon equation (TDNLSGE), since most of the research focused on the numerical solutions for this problem. The reduced differential transform method is used for this purpose for several reasons. The first reason is that the method has not previously been studied to solve this problem. Secondly, the present method is easy to apply for multidimensional problems and the corresponding algebraic equation is simple and easy to implement. Thirdly, this method can reduce the size of the calculations and can provide an analytic approximation, in many cases exact solutions, in rapidly convergent power series form with elegantly computed terms ([37] and see the references therein). Moreover, the reduced differential transform method (RDTM) has an alternative approach of solving problems to overcome the demerit of discretization, linearization, or perturbations of well-known numerical and analytical methods such as Adomian decomposition, differential transform, homotopy perturbation, and variational iteration [37-39].

In this paper, we investigate the solution of the twodimensional nonlinear sine-Gordon equation [40]:

$\frac{\partial^{2} u}{\partial t^{2}}+\beta \frac{\partial u}{\partial t}=\alpha\left(\frac{\partial^{2} u}{\partial x^{2}}+\frac{\partial^{2} u}{\partial y^{2}}\right)-\phi(x, y) \sin u+h(x, y, t),(x, y) \in \Omega, t \geq 0$,

subject to the initial conditions:

$$
\begin{gathered}
u(x, y, 0)=\varphi_{1}(x, y), x, y \in \Omega, \\
\frac{\partial}{\partial t} u(x, y, 0)=\varphi_{2}(x, y), x, y \in \Omega,
\end{gathered}
$$

by using RDTM, where $\Omega=\{(x, y): a \leq x \leq b, c \leq y \leq d\}$.

The function $\phi(x, y)$ can be interpreted as a Josephson current density, and $\varphi_{1}(x, y)$ and $\varphi_{2}(x, y)$ are wave modes or kinks and velocity, respectively. The parameter $\beta$ is the socalled dissipative term, which is assumed to be a real number with $\beta \geq 0$. When $\beta=0$, Equation (1) reduces to the undamped SGE equation in two space variables, while when $\beta>0$, to the damped one, and $\alpha$ is a nonnegative real number.

The paper is organized as follows. In Section 2, we begin with some basic definitions and operations of the proposed method, and we introduce some lemmas that will be used later in this paper. The implementation of the method is presented in Section 3. The convergence analysis of the method is presented in Section 4. In Section 5, we apply RDTM to solve four test problems to show the applicability, efficiency, and accuracy of the method. Section 6 presents graphical representation and physical interpretations of the solutions behavior of the considered examples. Conclusions are given in Section 7.

\section{Preliminaries and Notations}

In this section, we give the basic definitions and operations of the two-dimensional reduced differential transform method [37, 41-43].

Definition 1. If a function $u(x, y, t)$ is analytic and differentiated continuously with respect to space variables $x, y$ and time variable $t$ in the domain of interest, then

$$
U_{k}(x, y)=\frac{1}{k !}\left[\frac{\partial^{k}}{\partial t^{k}} u(x, y, t)\right]_{t=t_{0}},
$$

where $U_{k}(x, y)$ is the $t$-dimensional spectrum function or the transformed function.

Definition 2. The inverse reduced differential transform of a sequence $\left\{U_{k}(x, y)\right\}_{k=0}^{\infty}$ is given by

$$
u(x, y, t)=\sum_{k=0}^{\infty} U_{k}(x, y)\left(t-t_{0}\right)^{k}
$$

Then, combining Equations (4) and (5), we write

$$
u(x, y, t)=\sum_{k=0}^{\infty} \frac{1}{k !}\left[\frac{\partial^{k}}{\partial t^{k}} u(x, y, t)\right]_{t=t_{0}}\left(t-t_{0}\right)^{k}
$$

Remark 3. The function $u(x, y, t)$ is represented by a finite series (5) around $t_{0}=0$ and can be written as $\tilde{u}_{n}(x, y, t)=$ $\sum_{k=0}^{n} U_{k}(x, y) t^{k}+R_{n}(x, y, t)$ where the tail function $R_{n}(x, y, t$ )$=\sum_{k=n+1}^{\infty} U_{k}(x, y) t^{k}$ is negligibly small.

Furthermore, the inverse reduced differential transform of the set of $\left\{U_{k}(x, y)\right\}_{k=0}^{n}$ gives an approximate solution as

$$
\tilde{u}_{n}(x, y, t)=\sum_{k=0}^{n} U_{k}(x, y) t^{k}
$$

where $n$ is the order of the approximate solution. Therefore, by Definition 2, the exact solution of the problem is given by

$$
u(x, y, t)=\lim _{n \rightarrow \infty} \tilde{u}_{n}(x, y, t)=\sum_{k=0}^{\infty} U_{k}(x, y) t^{k} .
$$

From Equation (8), it can be found that the concept of the reduced differential transform method is derived from the power series expansion.

The fundamental mathematical operations performed by RDTM are listed in Table 1.

In addition to the properties of RDTM given in Table 1, we introduce the lemmas which provide us with a simple way to apply the RDTM to the two-dimensional nonlinear sine-Gordon Equations (1)-(3). 
TABLE 1: The fundamental mathematical operations of RDTM $[37,41,44]$.

\begin{tabular}{lr}
\hline Original function & Transformed function \\
\hline$u(x, y, t)$ & $U_{k}(x, y)=1 / k !\left[\left(\partial^{k} / \partial t^{k}\right) u(x, y, t)\right]_{t=0}$ \\
$w(x, y, t)=\alpha u(x, y, t) \pm \beta v(x, y, t)$ & $W_{k}(x, y)=\alpha U_{k}(x, y) \pm \beta V_{k}(x, y)$, where $\alpha$ and $\beta$ are constants \\
$w(x, y, t)=x^{m} y^{n} t^{p}$ & $W_{k}(x, y)=x^{m} y^{n} \delta(k-p)$, where $\delta(k-p)=\left\{\begin{array}{l}1, \text { if } k=p, \\
0, \text { if } k \neq p\end{array}\right.$ \\
$w(x, y, t)=x^{m} y^{n} t^{p} u(x, y, t)$ & $W_{k}(x, y)=x^{m} y^{n} U_{(k-p)}(x, y)$ \\
$w(x, y, t)=u(x, y, t) v(x, y, t)$ & $W_{k}(x, y)=\sum_{r=0}^{k} U_{k}(x, y) V_{k-r}(x, y)=\sum_{r=0}^{k} V_{k}(x, y) U_{k-r}(x, y)$ \\
$w(x, y, t)=\left(\partial^{r} / \partial t^{r}\right) u(x, y, t)$ & $W_{k}(x, y)=((k+r) ! / k !) U_{k+r}(x, y)=(k+1)(k+2) \cdots(k+r) U_{k+r}(x, y)$ \\
$w(x, y, t)=\left(\partial^{n} / \partial x^{n}\right) u(x, y, t)$ & $W_{k}(x, y)=\left(\partial^{n} / \partial x^{n}\right) U_{k}(x, y)$ \\
$w(x, y, t)=\left(\partial^{n} / \partial y^{n}\right) u(x, y, t)$ & $W_{k}(x, y)=\left(\partial^{n} / \partial y^{n}\right) U_{k}(x, y)$ \\
$w(x, y, t)=\sin (\alpha x+\beta y+\omega t)$ & $W_{k}(x, y)=\left(\omega^{k} / k !\right) \sin ((k \pi / 2 !)+\alpha x+\beta y)$, where $\alpha, \beta$, and $\omega$ are constants \\
$w(x, y, t)=\cos (\alpha x+\beta y+\omega t)$ & $W_{k}(x, y)=\left(\omega^{k} / k !\right) \cos ((k \pi / 2 !)+\alpha x+\beta y)$, where $\alpha, \beta$, and $\omega$ are constants \\
\hline
\end{tabular}

Lemma 4. Assume that $F_{k}(x, y), G_{k}(x, y)$ and $U_{k}(x, y)$ are the reduced differential transform of the functions $f(x, y, t), g(x$ $, y, t)$ and $u(x, y, t)$, respectively, then, we have the following RDTM results:

(i) If $f(x, y, t)=\sin u(x, y, t)$, then

(ii) If $g(x, y, t)=\cos u(x, y, t)$, then

$$
G_{k}(x, y)= \begin{cases}\cos U_{0}, & \text { if } k=0, \\ -\sum_{k_{1}=0}^{k-1}\left(1-\frac{k_{1}}{k}\right) F_{k_{1}}(x, y) U_{k-k_{1}}(x, y), & \text { if } k \geq 1\end{cases}
$$

Proof.

(i) Applying properties of RDTM on both sides of $f(x$, $y, t)=\sin u(x, y, t)$, , we obtain

$$
F_{0}(x, y)=\sin U_{0}(x, y) .
$$

Then, by Leibnitz rule for higher order derivatives of the products and properties of RDTM on $f(x, y, t)=\sin u(x, y, t)$,

we obtain,

$$
F_{k}(x, y)=\frac{1}{k !}\left[\frac{\partial^{k}}{\partial t^{k}} f(x, y, t)\right]_{t=0}=\frac{1}{k !}\left[\frac{\partial^{k}}{\partial t^{k}} \sin u(x, y, t)\right]_{t=0} .
$$

But $\left(\partial^{k} / \partial t^{k}\right) f(x, y, t)=\left(\partial^{k-1} / \partial t^{k-1}\right)(\cos u(x, y, t)(\partial / \partial t) u$ $(x, y, t))=\sum_{k_{1}=0}^{k-1}\left(\begin{array}{c}k-1 \\ k_{1}\end{array}\right)\left(\partial^{k_{1}} g(x, y, t) / \partial t^{k_{1}}\right)\left(\partial^{k-k_{1}} u(x, y, t) /\right.$ $\left.\partial t^{k-k_{1}}\right)$

Therefore,

$$
\begin{aligned}
{\left[\frac{\partial^{k}}{\partial t^{k}} f(x, y, t)\right]_{t=0} } & =\sum_{k_{1}=0}^{k-1}\left(\begin{array}{c}
k-1 \\
k_{1}
\end{array}\right) k_{1} !\left(k-k_{1}\right) ! G_{k_{1}}(x, y) U_{k-k_{1}}(x, y), \\
& =\sum_{k_{1}=0}^{k-1}(k-1) !\left(k-k_{1}\right) G_{k_{1}}(x, y) U_{k-k_{1}}(x, y) .
\end{aligned}
$$

Hence, by using Definition 1 , for $k=1,2,3, \cdots$, we get

$$
\begin{aligned}
F_{k}(x, y) & =\frac{1}{k !}\left[\frac{\partial^{k}}{\partial t^{k}} f(x, y, t)\right]_{t=0} \\
& =\sum_{k_{1}=0}^{k-1} \frac{1}{k !}(k-1) !\left(k-k_{1}\right) G_{k_{1}}(x, y) U_{k-k_{1}}(x, y) \\
& =\sum_{k_{1}=0}^{k-1}\left(1-\frac{k_{1}}{k}\right) G_{k_{1}}(x, y) U_{k-k_{1}}(x, y)
\end{aligned}
$$

(ii) Applying properties of RDTM on both sides of $g(x$ $, y, t)=\cos u(x, y, t)$, we get 


$$
G_{0}(x, y)=\cos U_{0}(x, y) .
$$

Using Leibnitz rule of higher order derivatives of the products on $g(x, y, t)=\cos u(x, y, t)$, we get

$$
\begin{aligned}
& G_{k}(x, y)=\frac{1}{k !}\left[\frac{\partial^{k}}{\partial t^{k}} g(x, y, t)\right]_{t=0}=\frac{1}{k !}\left[\frac{\partial^{k}}{\partial t^{k}} \cos u(x, y, t)\right]_{t=0}, \\
& \frac{\partial^{k}}{\partial t^{k}} g(x, y, t)=-\frac{\partial^{k-1}}{\partial t^{k-1}}\left(\sin u(x, y, t) \frac{\partial}{\partial t} u(x, y, t)\right) \\
&=-\sum_{k_{1}=0}^{k-1}\left(\begin{array}{c}
k-1 \\
k_{1}
\end{array}\right) \frac{\partial^{k_{1}} f(x, y, t)}{\partial t^{k_{1}}} \frac{\partial^{k-k_{1}} u(x, y, t)}{\partial t^{k-k_{1}}} .
\end{aligned}
$$

Therefore,

$$
\begin{aligned}
{\left[\frac{\partial^{k}}{\partial t^{k}} g(x, y, t)\right]_{t=0} } & =-\sum_{k_{1}=0}^{k-1}\left(\begin{array}{c}
k-1 \\
k_{1}
\end{array}\right) k_{1} !\left(k-k_{1}\right) ! F_{k_{1}}(x, y) U_{k-k_{1}}(x, y), \\
& =-\sum_{k_{1}=0}^{k-1}(k-1) !\left(k-k_{1}\right) F_{k_{1}}(x, y) U_{k-k_{1}}(x, y)
\end{aligned}
$$

and then using Definition 1 , for $k=1,2,3, \cdots$, we get

$$
\begin{aligned}
G_{k}(x, y) & =\frac{1}{k !}\left[\frac{\partial^{k}}{\partial t^{k}} g(x, y, t)\right]_{t=0} \\
& =\sum_{k_{1}=0}^{k-1} \frac{1}{k !}(k-1) !\left(k-k_{1}\right) F_{k_{1}}(x, y) U_{k-k_{1}}(x, y) \\
& =-\sum_{k_{1}=0}^{k-1}\left(1-\frac{k_{1}}{k}\right) F_{k_{1}}(x, y) U_{k-k_{1}}(x, y)
\end{aligned}
$$

Lemma 5. If $\left(\partial^{k} u(x, y, t)\right) /\left(\partial t^{k}\right)=\varphi(x, y)$, then

$$
U_{k}(x, y)=\frac{\varphi(x, y)}{k !}
$$

Proof. By Definition 1, $U_{k}(x, y)=1 / k !\left[\left(\partial^{k} u(x, y, t)\right) /\left(\partial t^{k}\right)\right]_{t=0}$, and so, when $k$ is replaced by $n$, we have,

$$
U_{n}(x, y)=\frac{1}{n !}\left[\frac{\partial^{n} u(x, y, t)}{\partial t^{n}}\right]_{t=0},
$$

\begin{tabular}{|c|c|c|c|c|c|}
\hline$x$ & $y$ & $t$ & $u_{8}$ & Exact & $\mid u_{8}-$ Exact $\mid$ \\
\hline \multirow{10}{*}{1} & \multirow{10}{*}{1} & 0.1 & 0.9950 & 0.9950 & $1.1102 \times 10^{-16}$ \\
\hline & & 0.2 & 0.9801 & 0.9801 & $2.8200 \times 10^{-14}$ \\
\hline & & 0.3 & 0.9553 & 0.9553 & $1.6261 \times 10^{-12}$ \\
\hline & & 0.4 & 0.9211 & 0.9211 & $2.8861 \times 10^{-11}$ \\
\hline & & 0.5 & 0.8776 & 0.8776 & $2.6861 \times 10^{-10}$ \\
\hline & & 0.6 & 0.8253 & 0.8253 & $1.6618 \times 10^{-09}$ \\
\hline & & 0.7 & 0.7648 & 0.7648 & $7.7554 \times 10^{-09}$ \\
\hline & & 0.8 & 0.6967 & 0.6967 & $2.9446 \times 10^{-08}$ \\
\hline & & 0.9 & 0.6216 & 0.6216 & $9.5499 \times 10^{-08}$ \\
\hline & & 1 & 0.5403 & 0.5403 & $2.7350 \times 10^{-07}$ \\
\hline
\end{tabular}

and from the initial condition we get $\left(\partial^{n} u(x, y, t)\right) /\left(\partial t^{n}\right)=$ $=\varphi(x, y)$.

Thus, $U_{n}(x, y)=1 / n !\left[\left(\partial^{n} u(x, y, t)\right) /\left(\partial t^{n}\right)\right]_{t=0}=1 / n ![\varphi(x$, $y)]_{t=0}=\varphi(x, y) / n !$.

Therefore, $U_{k}(x, y)=\varphi(x, y) / k !$

Furthermore, by convention, if $u(x, y, 0)=\varphi_{1}(x, y)$, then $U_{0}(x, y)=\varphi_{1}(x, y) / 0 !=\varphi_{1}(x, y)$ and if $\partial / \partial t u(x, y, 0)=\varphi_{2}(x$, $y)$, then $U_{1}(x, y)=\varphi_{2}(x, y) / 1 !=\varphi_{2}(x, y)$.
TABLE 2: Eighth-order approximate solution by RDTM of Example 1 for different values of $t$ and comparison with the exact solution.

\section{Implementation of the Method}

To illustrate the basic concepts of the RDTM, we consider the NLSGE (1) with initial conditions (2) and (3).

According to the RDTM given in Table 1 and Lemma 4, we can construct the following iteration formula:

$$
\begin{aligned}
(k & +2)(k+1) U_{k+2}(x, y)+\beta(k+1) U_{k+1}(x, y) \\
& =\alpha\left(\frac{\partial^{2}}{\partial x^{2}} U_{k}(x, y)+\frac{\partial^{2}}{\partial y^{2}} U_{k}(x, y)\right)-\phi(x, y) F_{k}(x, y)+H_{k}(x, y),
\end{aligned}
$$

where $F_{k}(x, y)$ is the reduced differential transform of the nonlinear term $\sin u(x, y, t)$ and $H_{k}(x, y)$ is the reduced differential transform of the inhomogeneous term $h(x, y, t)$.

Thus,

$$
F_{0}(x, y)=\sin U_{0},
$$

$$
\begin{gathered}
F_{1}(x, y)=G_{0}(x, y) U_{1}(x, y), \\
F_{2}(x, y)=G_{0}(x, y) U_{2}(x, y)-\frac{1}{2} F_{0}(x, y) U_{1}^{2}(x, y), \\
F_{3}(x, y)=G_{0}(x, y) U_{3}(x, y)-F_{0}(x, y) U_{1}(x, y) U_{2}(x, y) \\
-\frac{1}{6} G_{0}(x, y) U_{1}^{3}(x, y), \\
F_{4}(x, y)=G_{0}(x, y) U_{4}(x, y)-F_{0}(x, y) U_{1}(x, y) U_{3}(x, y) \\
-\frac{1}{2} F_{0}(x, y) U_{2}^{2}(x, y)-\frac{1}{2} G_{0}(x, y) U_{1}^{2}(x, y) U_{2}(x, y) \\
+\frac{1}{24} F_{0}(x, y) U_{1}^{4}(x, y),
\end{gathered}
$$

and so on. 


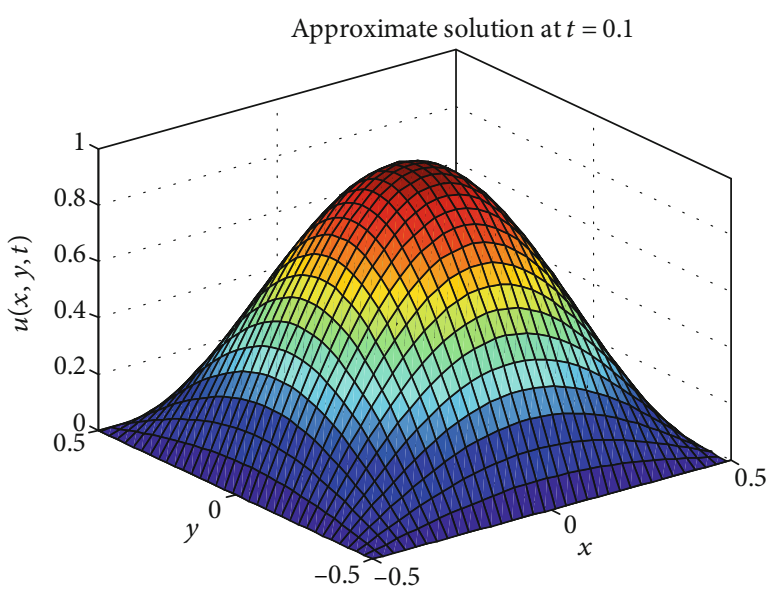

(a)

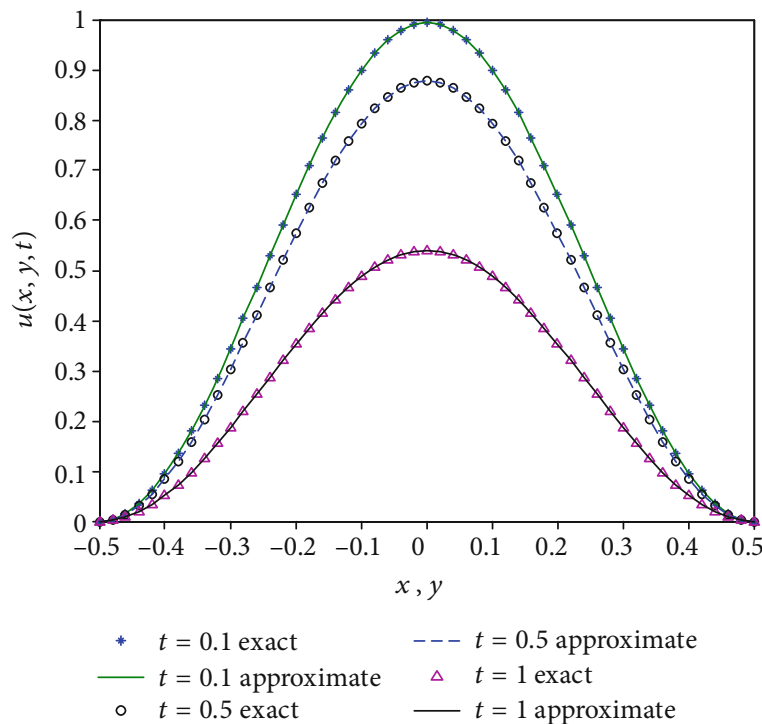

(c)

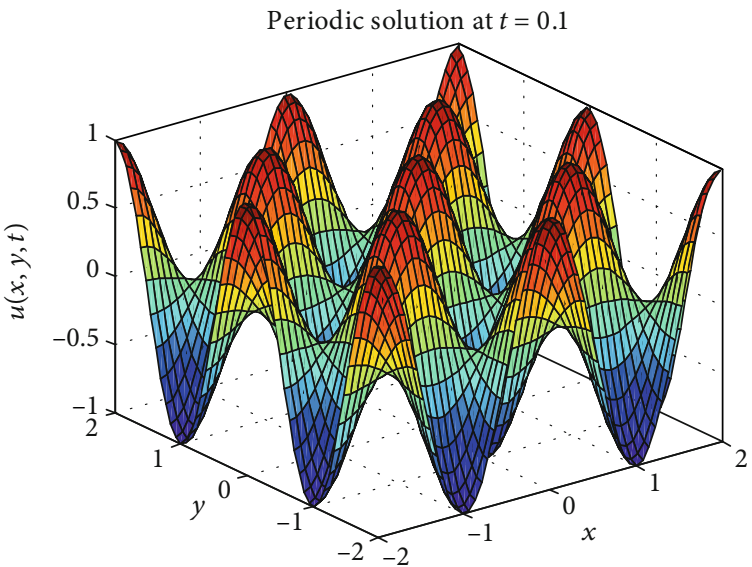

(e)

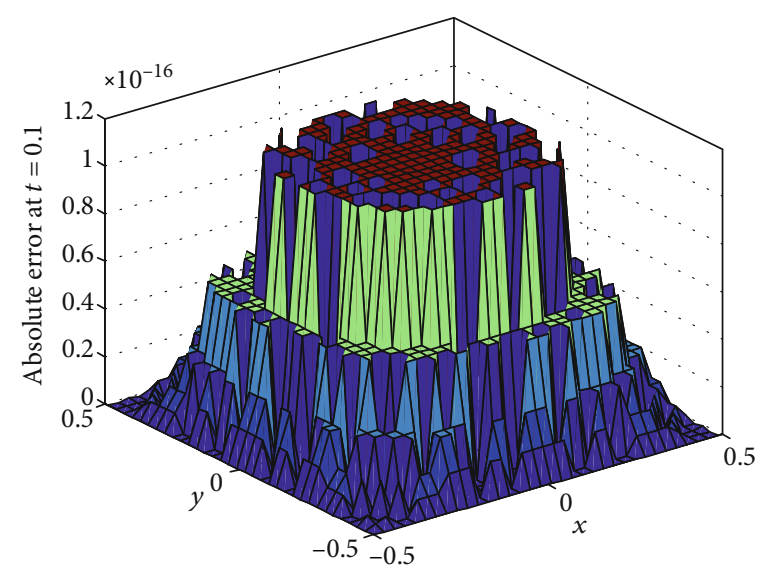

(b)

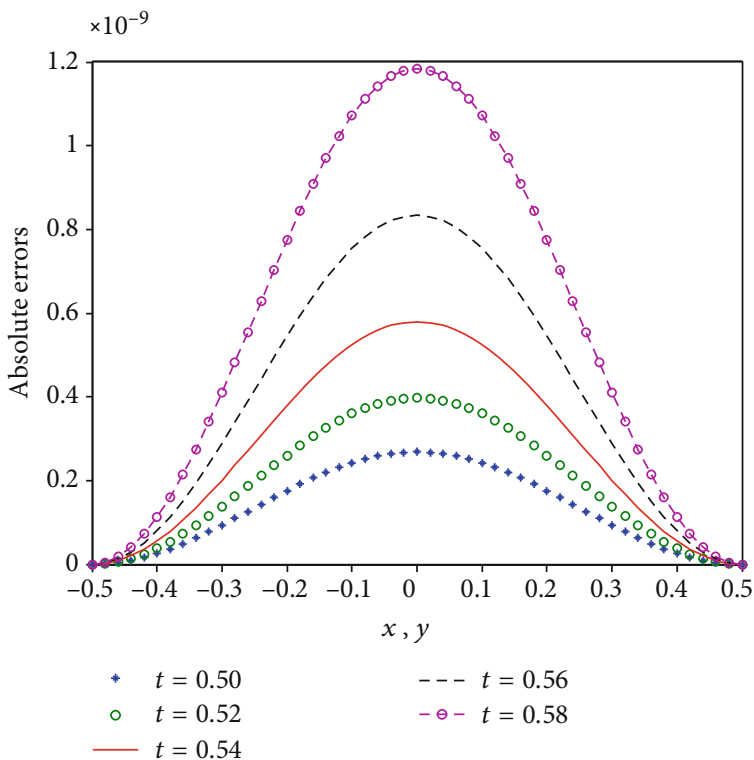

(d)

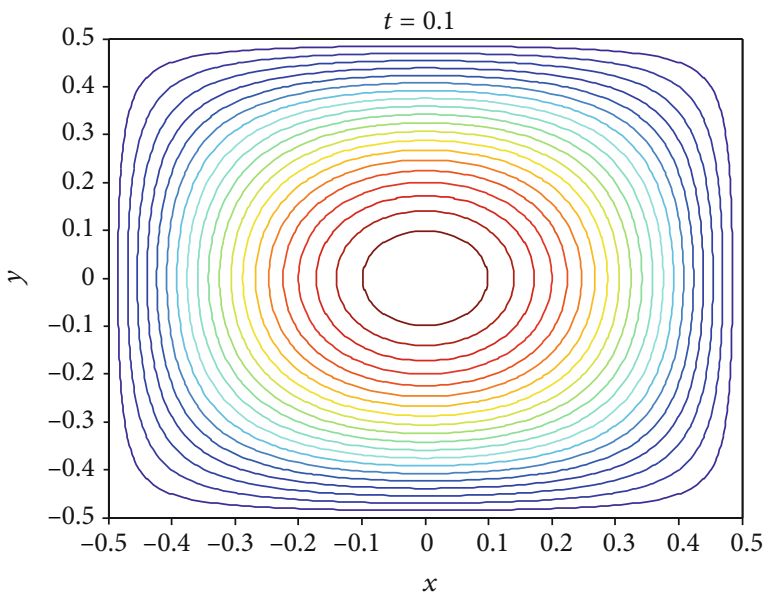

(f)

FIGURE 1: Plots of the solution behavior of Example 1: (a) approximated solutions at time $t=0.1$; (b) absolute errors at time $t=0.1$; (c) comparison of exact and approximated solutions at times $t=0.1,0.5,1 ;$ (d) comparison of absolute errors for different values of times $t=$ $0.5,0.52,0.54,0.56,0.58$; (e) the periodic nature of the solution; and (f) the soliton at $t=0.1$. 
Using Lemma 5 on initial conditions (2) and (3), we get

$$
\begin{gathered}
U_{0}(x, y)=\varphi_{1}(x, y), \\
U_{1}(x, y)=\varphi_{2}(x, y) .
\end{gathered}
$$

Substituting (24) and (23) into (22) and by straightforward iterative calculations, we get the following successive values of $U_{k}(x, y)$, i.e., $U_{2}(x, y), U_{3}(x, y), U_{4}(x, y), \cdots$. Then, the inverse reduced differential transform of the set of values $\left\{U_{k}(x, y)\right\}_{k=0}^{n}$ gives the $n$-term approximate solution:

$$
\tilde{u}_{n}(x, y, t)=\sum_{k=0}^{n} U_{k}(x, y) t^{k}
$$

Therefore, the exact solution of problem (1) is given by

$$
u(x, y, t)=\lim _{n \rightarrow \infty} \tilde{u}_{n}(x, y, t)=\sum_{k=0}^{\infty} U_{k}(x, y) t^{k}
$$

\section{Convergence Analysis}

In this section, we present the convergence analysis of the approximate analytical solutions which are computed from the application of RDTM [41].

Consider the SGE (1) in the following functional equation form:

$$
u(x, y, t)=\mathscr{F}(u(x, y, t))
$$

where $\mathscr{F}$ is a general nonlinear operator involving both linear and nonlinear terms.

According to RDTM, the two-dimensional NLSGE given in Equation (1) has a solution of the form:

$$
u(x, y, t)=\sum_{k=0}^{\infty} U_{k}(x, y) t^{k}=\sum_{k=0}^{\infty} \beta_{k} .
$$

It is noted that the solutions by RDTM is equivalent to determining the sequences

$$
\begin{gathered}
S_{0}=U_{0}(x, y)=\beta_{0}, \\
S_{1}=U_{0}(x, y)+U_{1}(x, y) t=\beta_{0}+\beta_{1}, \\
S_{2}=U_{0}(x, y)+U_{1}(x, y) t+U_{2}(x, y) t^{2}=\beta_{0}+\beta_{1}+\beta_{2}, \\
\ldots \\
S_{n}=\sum_{k=0}^{n} U_{k}(x, y) t^{k}=\sum_{k=0}^{n} \beta_{k},
\end{gathered}
$$

\begin{tabular}{|c|c|c|c|c|c|}
\hline$x$ & $y$ & $t$ & $u_{8}$ & Exact solution & $\mid u_{8}-$ Exact $\mid$ \\
\hline \multirow{10}{*}{1} & \multirow{10}{*}{1} & 0.1 & 3.6193 & 3.6193 & $1.1546 \times 10^{-14}$ \\
\hline & & 0.2 & 3.2749 & 3.2749 & $5.5334 \times 10^{-12}$ \\
\hline & & 0.3 & 2.9633 & 2.9633 & $2.1063 \times 10^{-10}$ \\
\hline & & 0.4 & 2.6813 & 2.6813 & $2.7781 \times 10^{-09}$ \\
\hline & & 0.5 & 2.4261 & 2.4261 & $2.0500 \times 10^{-08}$ \\
\hline & & 0.6 & 2.1952 & 2.1952 & $1.0477 \times 10^{-07}$ \\
\hline & & 0.7 & 1.9863 & 1.9863 & $4.1555 \times 10^{-07}$ \\
\hline & & 0.8 & 1.7973 & 1.7973 & $1.3692 \times 10^{-06}$ \\
\hline & & 0.9 & 1.6263 & 1.6263 & $3.9154 \times 10^{-06}$ \\
\hline & & 1 & 1.4715 & 1.4715 & $1.0013 \times 10^{-05}$ \\
\hline
\end{tabular}

by using the iterative scheme

$$
S_{n+1}=\mathscr{F}\left(S_{n}\right) \text {, }
$$

TABLE 3: Eighth-order approximate solution by RDTM of Example 2 for different values of $t$ and comparison with the exact solution.

associated with the functional equation

$$
S=\mathscr{F}(S)
$$

Hence, the solution obtained by RDTM, $u(x, y, t)=$ $\sum_{k=0}^{\infty} U_{k}(x, y) t^{k}=\sum_{k=0}^{\infty} \beta_{k}$ is equivalent to

$$
\begin{aligned}
u(x, y, t) & =U_{0}(x, y)+U_{1}(x, y) t+U_{2}(x, y) t^{2}+U_{3}(x, y) t^{3}+U_{4}(x, y) t^{4}+\cdots \\
& =\left\{S_{n}\right\}_{n=0}^{\infty} .
\end{aligned}
$$

The sufficient condition for convergence of the series solution $\left\{S_{n}\right\}_{n=0}^{\infty}$ is given in the following theorems.

Theorem 6. Let $\mathscr{F}$ be an operator from a from Hilbert space $\mathscr{H}$ in to $\mathscr{H}$. Then, the series solution $\left\{S_{n}\right\}_{n=0}^{\infty}$ converges whenever there is $\alpha$ such that $0<\alpha<1$, and $\left\|\beta_{k+1}\right\| \leq \alpha\left\|\beta_{k}\right\|$.

See [41] for the proof.

Theorem 7. Let $\mathscr{F}$ be a nonlinear operator that satisfies the Lipschitz condition from Hilbert space $\mathscr{H}$ in to $\mathscr{H}$ and $u(x, y$ $, t)$ be the exact solution of the given SGE. If the series solution $\left\{S_{n}\right\}_{n=0}^{\infty}$ converges, then it converges to $u(x, y, t)$.

For proof see Ref. [41].

Definition 8. For $k \in N \cup\{0\}$, we define

$$
\begin{cases}\frac{\left\|\beta_{k+1}\right\|}{\left\|\beta_{k}\right\|}=\frac{\left\|U_{k+1}(x, y) t^{k+1}\right\|}{\left\|U_{k}(x, y) t^{k}\right\|}, & \text { if }\left\|\beta_{k}\right\|=\left\|U_{k}(x, y) t^{k}\right\| \neq 0, \\ 0, & \text { if }\left\|\beta_{k}\right\|=\left\|U_{k}(x, y) t^{k}\right\|=0 .\end{cases}
$$

Then, we can say that the series approximate solution $\left\{S_{n}\right\}_{n=0}^{\infty}$ converges to the exact solution $u(x, y, t)$ when $0 \leq$ $\alpha_{k}<1$ for $k=0,1,2, \cdots$. 


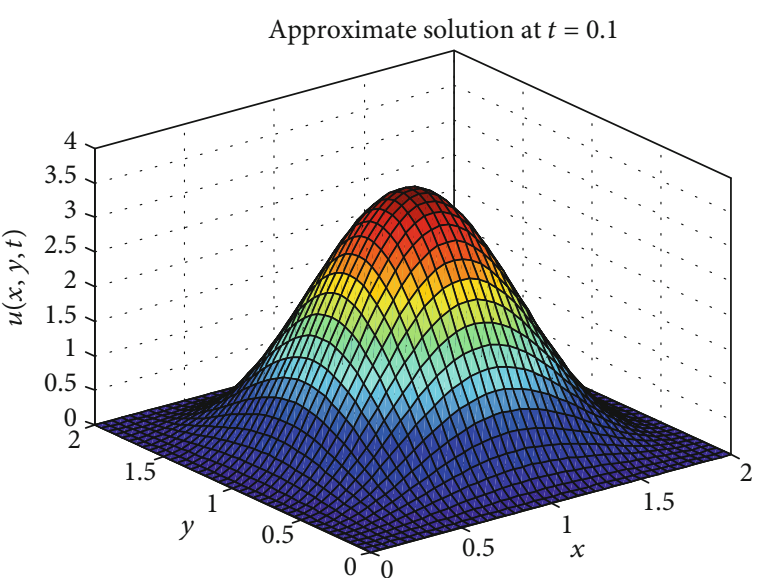

(a)
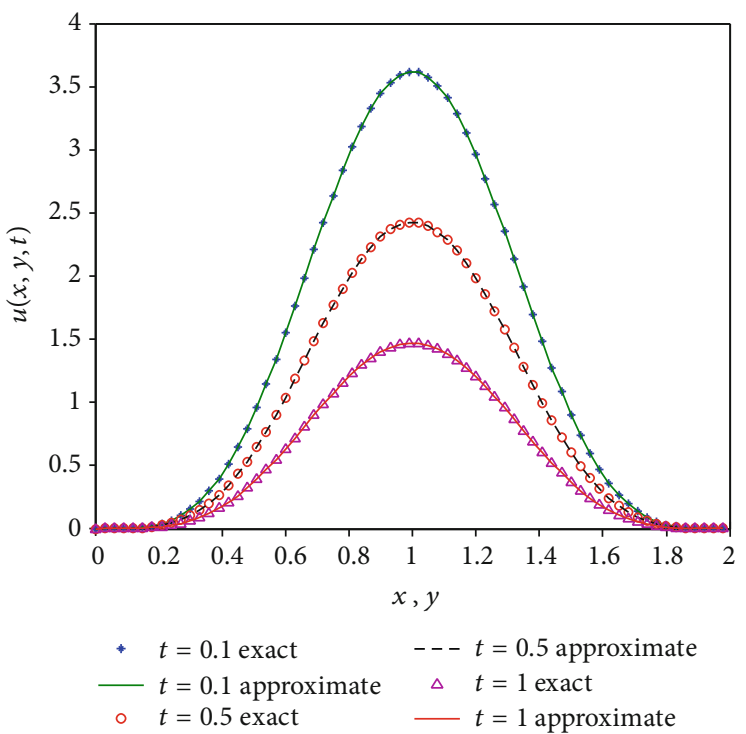

(c)

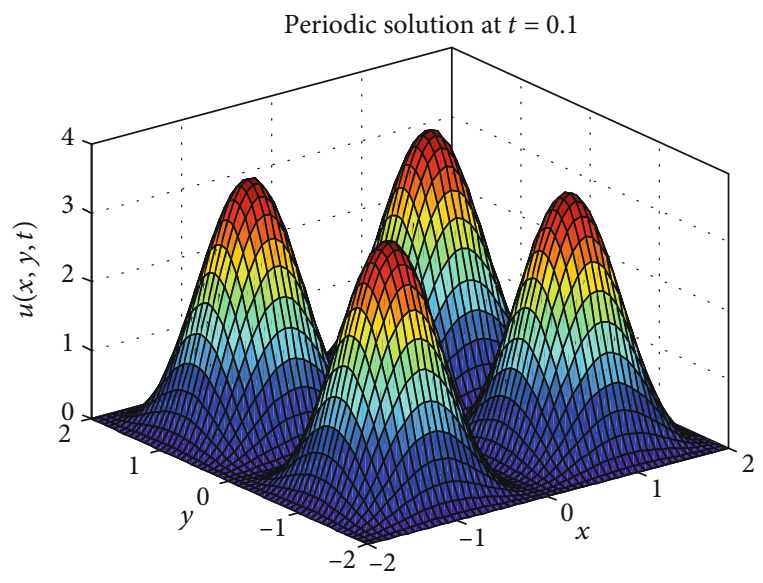

(e)

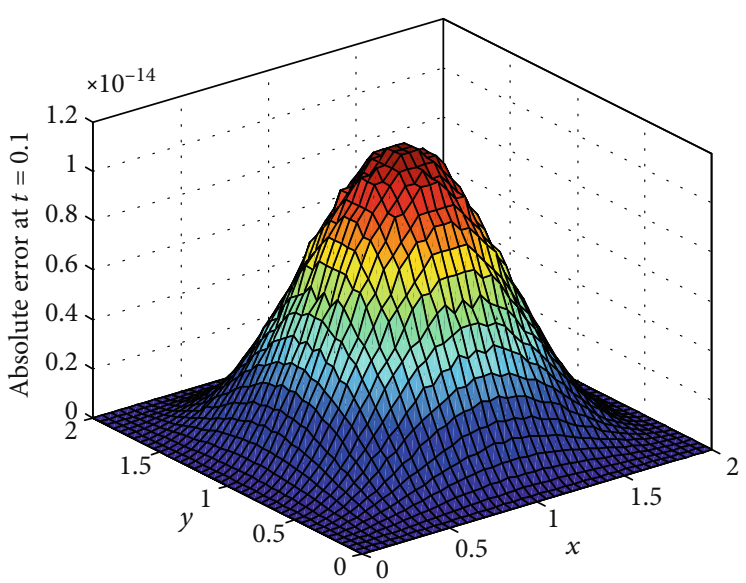

(b)

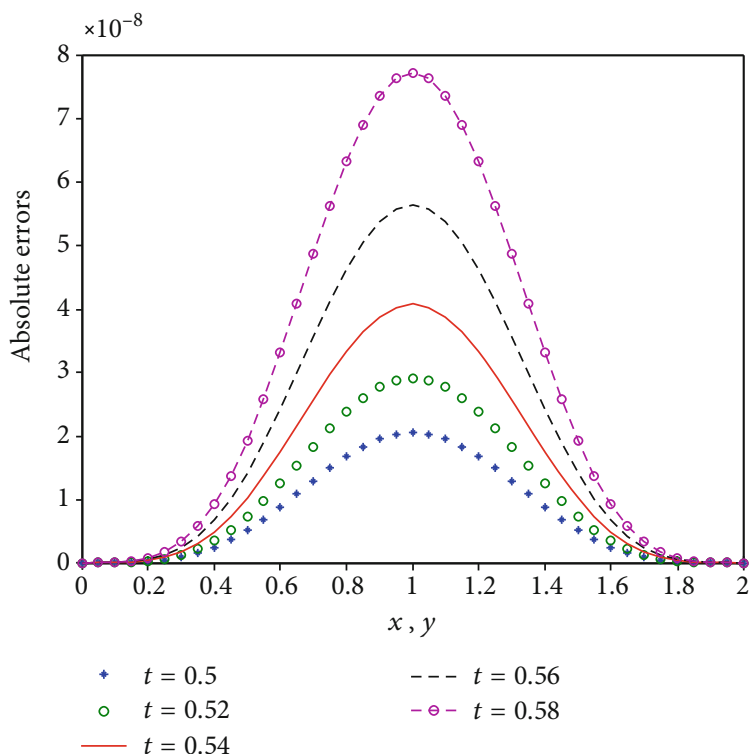

(d)

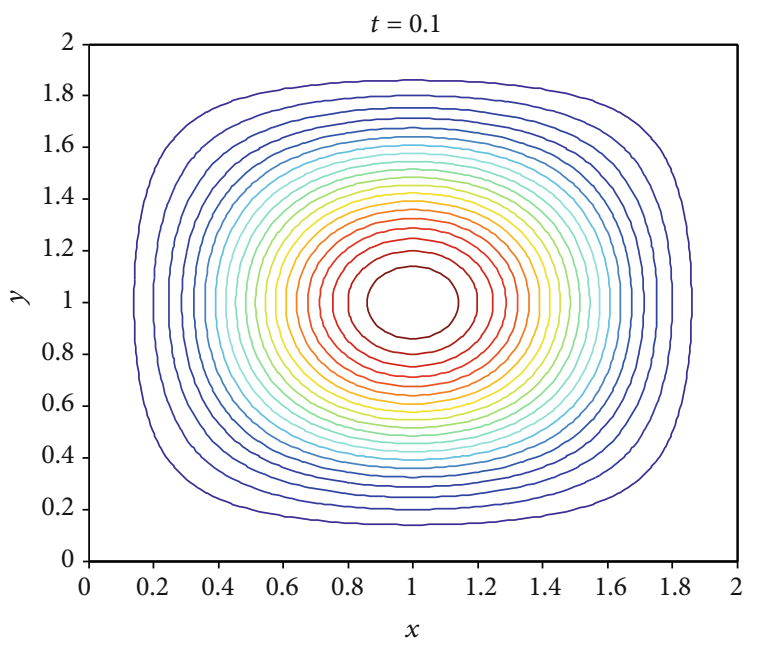

(f)

Figure 2: Plots of the solution behavior of Example 2: (a) approximated solutions at $t=0.1$; (b) absolute errors at time at $t=0.1$; (c) comparison of exact and approximated solutions for times $t=0.1,0.5,1$; (d) comparison of absolute errors for different values of times $t$ $=0.5,0.52,0.54,0.56,0.58$; (e) the periodic nature of the solution; and (f) the soliton at $t=0.1$. 
TABLE 4: Eighth-order approximate numerical solution by RDTM of Example 3 for different values of $t$ and comparison with the exact solution.

\begin{tabular}{|c|c|c|c|c|c|}
\hline$x$ & $y$ & $t$ & $u_{8}$ & Exact solution & $\mid u_{8}-$ Exact $\mid$ \\
\hline \multirow{10}{*}{1} & \multirow{10}{*}{1} & 0.1 & 0.8632 & 0.8632 & $1.1102 \times 10^{-16}$ \\
\hline & & 0.2 & 0.8085 & 0.8085 & $2.5535 \times 10^{-14}$ \\
\hline & & 0.3 & 0.7457 & 0.7457 & $1.4601 \times 10^{-12}$ \\
\hline & & 0.4 & 0.6755 & 0.6755 & $2.5806 \times 10^{-11}$ \\
\hline & & 0.5 & 0.5985 & 0.5985 & $2.3916 \times 10^{-10}$ \\
\hline & & 0.6 & 0.5155 & 0.5155 & $1.4733 \times 10^{-09}$ \\
\hline & & 0.7 & 0.4274 & 0.4274 & $6.8465 \times 10^{-09}$ \\
\hline & & 0.8 & 0.3350 & 0.3350 & $2.5884 \times 10^{-08}$ \\
\hline & & 0.9 & 0.2392 & 0.2392 & $8.3583 \times 10^{-08}$ \\
\hline & & 1 & 0.1411 & 0.1411 & $2.3833 \times 10^{-07}$ \\
\hline
\end{tabular}

\section{Numerical Results}

In this section, we apply the reduced differential transform method (RDTM) for finding the approximate analytic solutions of four test examples associated with the nonlinear sine-Gordon equations (NLSGEs) in a two-dimensional space. To demonstrate the applicability of the method and accuracy of the solutions, the results obtained by the proposed method is compared with the exact solution existing in the literature, and the numerical results and the absolute errors are given using tables and figures.

Example 1. Consider the sine-Gordon equation [40]

$$
\begin{aligned}
\frac{\partial^{2} u}{\partial t^{2}} & =\frac{1}{2 \pi^{2}}\left[\frac{\partial^{2} u}{\partial x^{2}}+\frac{\partial^{2} u}{\partial y^{2}}\right]-\sin u+\sin (\cos (\pi x) \cos (\pi y) \cos (t)),(x, y) \in\left[\frac{-1}{2}, \frac{1}{2}\right]^{2}, t \\
& \geq 0
\end{aligned}
$$

with initial conditions

$$
\begin{gathered}
u(x, y, 0)=\cos (\pi x) \cos (\pi y), \\
\frac{\partial}{\partial t} u(x, y, 0)=0 .
\end{gathered}
$$

Applying properties of RDTM to Equation (34), we construct the following recursive formula:

$$
\begin{aligned}
(k+2)(k+1) U_{k+2}(x, y)= & \frac{1}{2 \pi^{2}}\left[\frac{\partial^{2}}{\partial x^{2}} U_{k}(x, y)+\frac{\partial^{2}}{\partial y^{2}} U_{k}(x, y)\right] \\
& -F_{k}(x, y)+H_{k}(x, y),
\end{aligned}
$$

where $F_{k}(x, y)$ and $H_{k}(x, y)$ are the reduced differential transform of the nonlinear term $\sin (u(x, y, t))$ and the inhomogeneous term $\sin (\cos (\pi x) \cos (\pi y) \cos (t))$, respectively.
Using RDTM to the initial conditions (35) and (36), we get

$$
\begin{gathered}
U_{0}(x, y)=\cos (\pi x) \cos (\pi y), \\
U_{1}(x, y)=0 .
\end{gathered}
$$

Now taking the values of $k(k=0,1,2, \cdots)$, and applying Lemma 4 and using Equations (38) and (39) into Equation (37), we obtain the following successive iterated values:

$$
\begin{gathered}
U_{2}=-\frac{1}{2 !}[\cos (\pi x) \cos (\pi y)], \\
U_{3}=0, \\
U_{4}=\frac{1}{4 !}[\cos (\pi x) \cos (\pi y)], \\
U_{5}=0, \\
U_{6}=\frac{1}{6 !}[\cos (\pi x) \cos (\pi y)],
\end{gathered}
$$

and so on.

Then, by (8), we get

$u(x, y, t)=\sum_{k=0}^{\infty} U_{k}(x, y) t^{k}=\cos (\pi x) \cos (\pi y)\left(1-\frac{t^{2}}{2 !}+\frac{t^{4}}{4 !}-\frac{t^{6}}{6 !}+\cdots\right)$.

Hence, the exact solution of Example 1 is $u(x, y, t)=\cos$ $(\pi x) \cos (\pi y) \cos (t)$ as in Kang et al. [40].

For the convergence of the approximate analytic solution given in Equation (41), we calculate $\alpha_{k}$ using

$$
\alpha_{k}= \begin{cases}\frac{\left\|\beta_{k+1}\right\|}{\left\|\beta_{k}\right\|}, & \text { if }\left\|\beta_{k}\right\| \neq 0, \\ 0, & \text { if }\left\|\beta_{k}\right\|=0 .\end{cases}
$$

Hence, for $x, y \in[-1 / 2,1 / 2]$ and $t \geq 0$, we obtain $\alpha_{0}=0<1$ , $\alpha_{1}=0<1, \alpha_{2}=0<1, \alpha_{3}=0<1, \alpha_{4}=0<1, \cdots$, and by induction $\alpha_{k}<1$ for all $k \in N \cup\{0\}$. Therefore, using Definition 8, the solution of Equation (34) converges to the exact solution.

Numerical results corresponding to the two-dimensional nonlinear sine-Gordon equation given in Example 1 are depicted in Table 2 and Figure 1.

Example 2. Consider the two dimensional sine-Gordon equation $[36,45]$

$$
\begin{aligned}
\frac{\partial^{2} u}{\partial t^{2}}+\frac{\partial u}{\partial t}= & \frac{\partial^{2} u}{\partial x^{2}}+\frac{\partial^{2} u}{\partial y^{2}}-2 \sin u+2 \sin \left[e^{-t}(1-\cos (\pi x))(1-\cos (\pi y))\right], \\
& -\pi^{2} e^{-t}[\cos (\pi x)+\cos (\pi y)-2 \cos (\pi x) \cos (\pi y)],(x, y) \\
= & {[0,2]^{2}, t \geq 0 }
\end{aligned}
$$

with initial conditions

$$
u(x, y, 0)=(1-\cos (\pi x))(1-\cos (\pi y))
$$




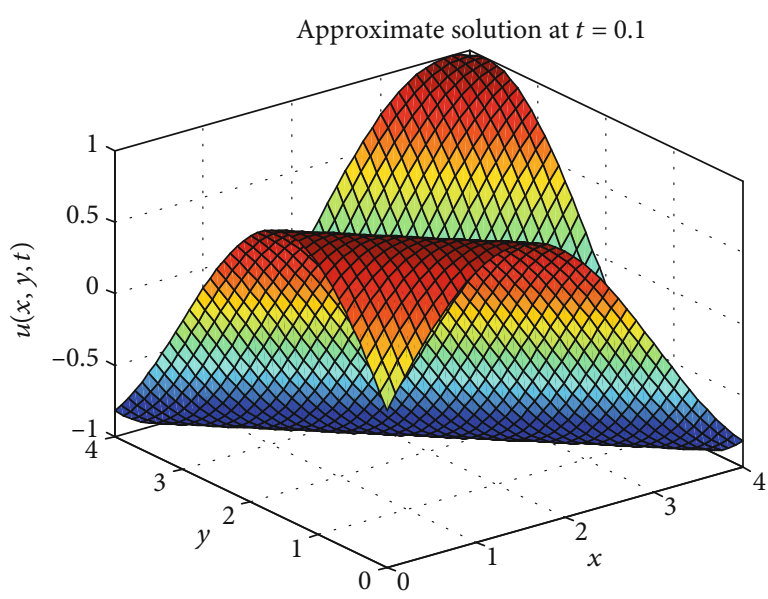

(a)

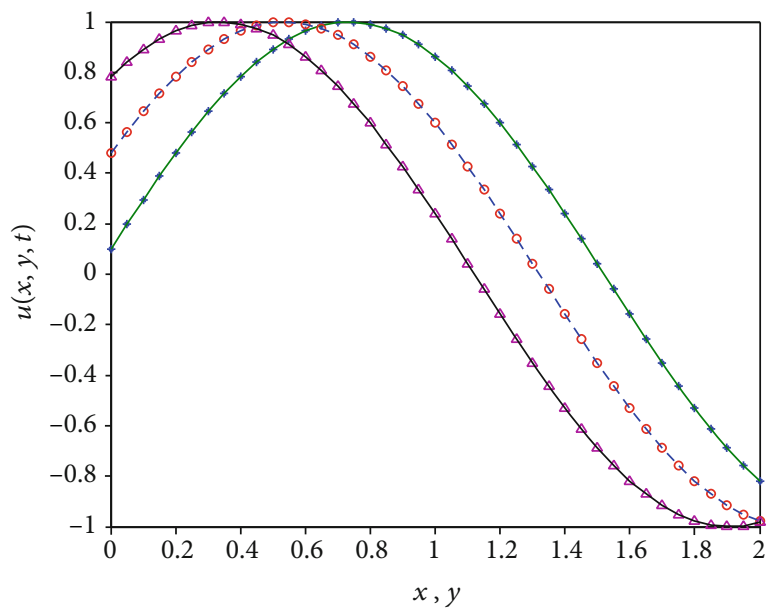

$$
\begin{aligned}
& +t=0.1 \text { exact }--t=0.5 \text { approximate } \\
& \text { - } t=0.1 \text { approximate } \Delta t=1 \text { exact } \\
& \circ t=0.5 \text { exact }-t=1 \text { approximate }
\end{aligned}
$$

(c)

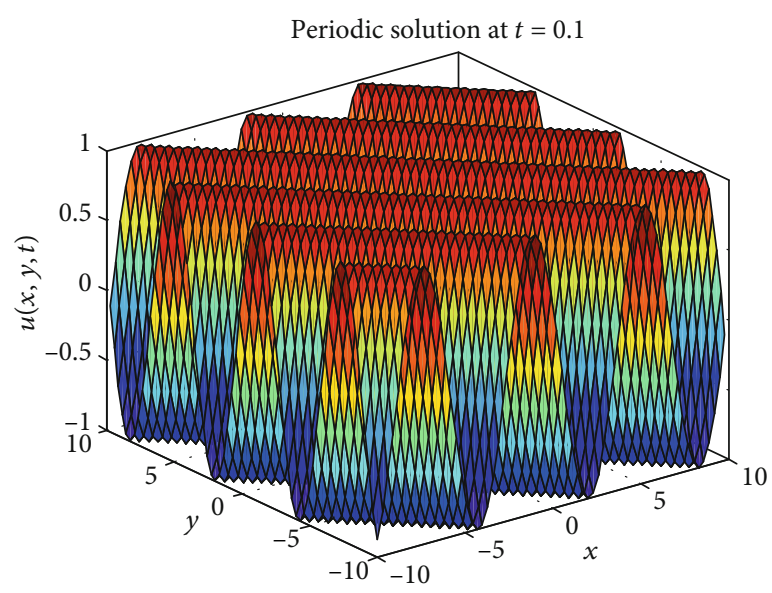

(e)

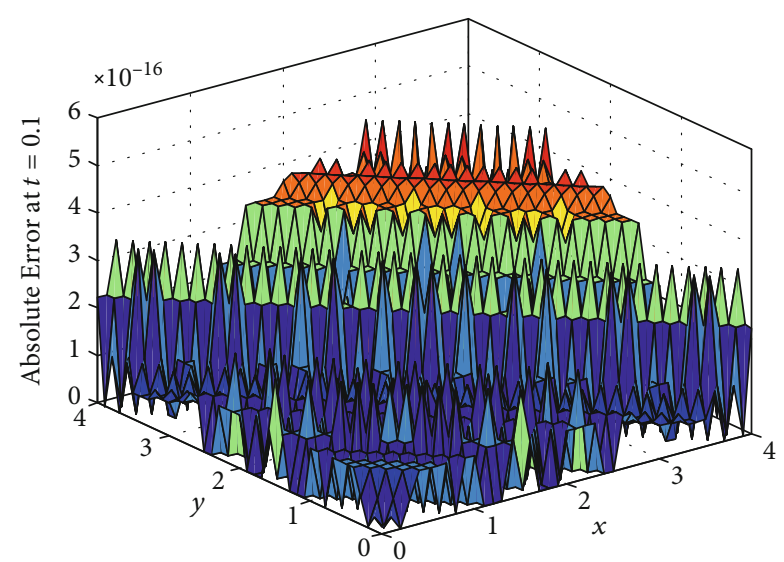

(b)

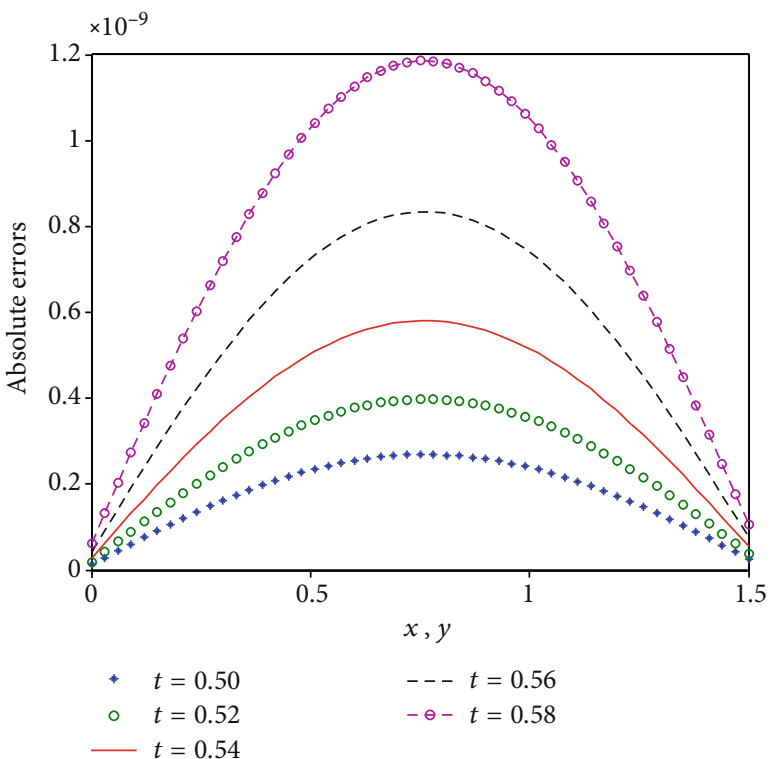

(d)

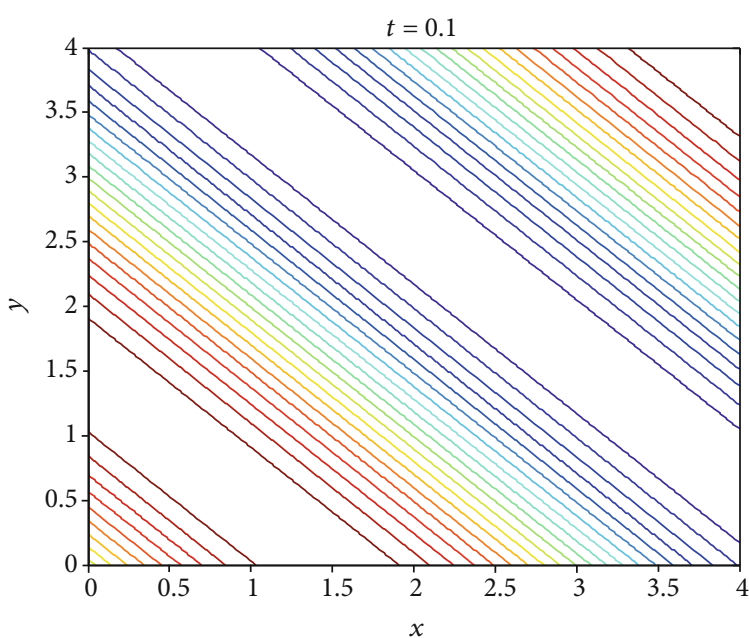

(f)

Figure 3: Plots of the solutions behavior of Example 3: (a) approximated solutions at $t=0.1$; (b) absolute errors $t=0.1$; (c) comparison of exact and approximated solutions for $t=0.1,0.5,1$; (d) comparison of absolute errors for different values of times $t=0.5,0.52,0.54,0.56$, 0.58 ; (e) the periodic nature of the solution, and (f) the soliton at $t=0.1$. 


$$
\frac{\partial}{\partial t} u(x, y, 0)=-(1-\cos (\pi x))(1-\cos (\pi y)) .
$$

By taking the reduced differential transform of Equation (43), we obtain

$$
\begin{aligned}
& (k+2)(k+1) U_{k+2}(x, y)+(k+1) U_{k+1}(x, y) \\
& =\frac{\partial^{2}}{\partial x^{2}} U_{k}(x, y)+\frac{\partial^{2}}{\partial y^{2}} U_{k}(x, y)-2 F_{k}(x, y)+H_{k}(x, y),
\end{aligned}
$$

where $F_{k}(x, y)$ and $H_{k}(x, y)$ are the reduced differential transform of the nonlinear term $\sin (u(x, y, t))$ and the inhomogeneous term

$$
\begin{aligned}
2 \sin & {\left[e^{-t}((1-\cos (\pi x))(1-\cos (\pi y)))\right] } \\
& -\pi e^{-t}[\cos (\pi x)+\cos (\pi y)-2 \cos (\pi x) \cos (\pi y)],
\end{aligned}
$$

respectively.

Using RDTM to the initial conditions (44) and (45), we get

$$
\begin{array}{r}
U_{0}(x, y)=(1-\cos (\pi x))(1-\cos (\pi y)), \\
U_{1}(x, y)=-(1-\cos (\pi x))(1-\cos (\pi y)) .
\end{array}
$$

Substituting Equations (48) and (49) into Equation (46), and applying Lemma 4, Definition 1, and properties of RDTM, we obtain the following successive iterated values for $k(k=0,1,2, \cdots)$ :

$$
\begin{aligned}
& U_{2}=\frac{1}{2 !}[(1-\cos (\pi x))(1-\cos (\pi y))], \\
& U_{3}=-\frac{1}{3 !}[(1-\cos (\pi x))(1-\cos (\pi y))], \\
& U_{4}=\frac{1}{4 !}[(1-\cos (\pi x))(1-\cos (\pi y))], \\
& U_{5}=-\frac{1}{5 !}[(1-\cos (\pi x))(1-\cos (\pi y))],
\end{aligned}
$$

and so on.

Then by (8), we obtain the approximate analytic solution

\begin{tabular}{|c|c|c|c|c|c|}
\hline$x$ & $y$ & $t$ & $u_{7}$ & Exact solution & $\mid u_{7}-$ Exact $\mid$ \\
\hline \multirow{10}{*}{1} & \multirow{10}{*}{1} & 0.1 & 5.6893 & 5.6893 & $2.3634 \times 10^{-12}$ \\
\hline & & 0.2 & 5.6279 & 5.6279 & $6.1136 \times 10^{-10}$ \\
\hline & & 0.3 & 5.5604 & 5.5604 & $1.5797 \times 10^{-08}$ \\
\hline & & 0.4 & 5.4863 & 5.4863 & $1.5875 \times 10^{-07}$ \\
\hline & & 0.5 & 5.4050 & 5.4050 & $9.4946 \times 10^{-07}$ \\
\hline & & 0.6 & 5.3161 & 5.3161 & $4.0838 \times 10^{-06}$ \\
\hline & & 0.7 & 5.2189 & 5.2189 & $1.3970 \times 10^{-05}$ \\
\hline & & 0.8 & 5.1129 & 5.1130 & $4.0343 \times 10^{-05}$ \\
\hline & & 0.9 & 4.9977 & 4.9978 & $1.0218 \times 10^{-04}$ \\
\hline & & 1 & 4.8729 & 4.8731 & $2.3289 \times 10^{-04}$ \\
\hline
\end{tabular}
of Example 2 as follows:

$$
\begin{aligned}
u(x, y, t)= & \sum_{k=0}^{\infty} U_{k}(x, y) t^{k}=(1-\cos (\pi x))(1-\cos (\pi y)) \\
& \cdot\left[1-t+\frac{1}{2 !} t^{2}-\frac{1}{3 !} t^{3}+\frac{1}{4 !} t^{4}-\frac{1}{5 !} t^{5}+\cdots\right] .
\end{aligned}
$$

The exact solution of the problem is $u(x, y, t)=e^{-t}(1-$ $\cos (\pi x))(1-\cos (\pi y))$, as indicated in $[36,45]$.

To test the convergence of the approximate solution, we calculate $\alpha_{k}$. Let us take $x=y=1$ and $t=0.5$ in the domain of interest, then using definition 8 , we obtain, $\alpha_{0}=0.5<1, \alpha_{1}$
TABLE 5: Seventh-order approximate solution by RDTM of Example 4 for different values of $t$ and comparison with the exact solution.

$=0.25<1, \alpha_{2}=0.1666666667<1, \alpha_{3}=0.125<1, \alpha_{4}=0.1<1$ ,...., and by induction $\alpha_{k}<1$ for all $k \in N \cup\{0\}$. Therefore, the solution of Equation (43) converges to the exact solution.

Numerical results corresponding to the two-dimensional nonlinear sine-Gordon equation given in Example 2 are depicted in Table 3 and Figure 2.

Example 3. Consider the two-dimensional inhomogeneous sine-Gordon equation [34],

$$
\begin{aligned}
\frac{\partial^{2} u}{\partial t^{2}}= & \frac{\partial^{2} u}{\partial x^{2}}+\frac{\partial^{2} u}{\partial y^{2}}-\sin u+\sin (\sin (x+y+t)) \\
& +\sin (x+y+t)(x, y) \in[0,2 \pi]^{2}, t \geq 0,
\end{aligned}
$$

with initial conditions

$$
\begin{array}{r}
u(x, y, 0)=\sin (x+y), \\
\frac{\partial u(x, y, 0)}{\partial t}=\cos (x+y) .
\end{array}
$$

Applying the RDTM to Equation (52), we obtain the following recurrence relation

$$
\begin{aligned}
(k+2)(k+1) U_{k+2}(x, y)= & \frac{\partial^{2}}{\partial x^{2}} U_{k}(x, y)+\frac{\partial^{2}}{\partial y^{2}} U_{k}(x, y) \\
& -F_{k}(x, y)+H_{k}(x, y)
\end{aligned}
$$

where $F_{k}(x, y)$ is the reduced differential transform of nonlinear term $\sin u(x, y, t)$ and $H_{k}(x, y)$ is the reduced differential transform of inhomogeneous term $[\sin (\sin (x$ $+y+t))+\sin (x+y+t)]$.

Using RDTM to the initial conditions (53) and (54), we have

$$
\begin{aligned}
& U_{0}(x, y)=\sin (x+y), \\
& U_{1}(x, y)=\cos (x+y) .
\end{aligned}
$$


Now taking the values of $k(k=0,1,2, \cdots)$ and applying Lemma 4, Definition 1, and properties of RDTM in Equation (55), we obtain the following successive iterative values:

$$
\begin{aligned}
& U_{2}=-\frac{1}{2 !} \sin (x+y), \\
& U_{3}=-\frac{1}{3 !} \cos (x+y), \\
& U_{4}=\frac{1}{4 !} \sin (x+y), \\
& U_{5}=\frac{1}{5 !} \cos (x+y), \\
& U_{6}=-\frac{1}{6 !} \sin (x+y), \\
& U_{7}=-\frac{1}{7 !} \cos (x+y),
\end{aligned}
$$

and so on.

Then, using Equation (8), we get

$$
\begin{aligned}
u(x, y, t)= & \sum_{k=0}^{\infty} U_{k}(x, y) t^{k}=\sin (x+y)\left(1-\frac{t^{2}}{2 !}+\frac{t^{4}}{4 !}-\frac{t^{6}}{6 !}+\cdots\right) \\
& +\cos (x+y)\left(t-\frac{t^{3}}{3 !}+\frac{t^{5}}{5 !}-\frac{t^{7}}{7 !}+\cdots\right) .
\end{aligned}
$$

Equation (58) represents the approximate analytic solution of Example 3, whose exact solution is $u(x, y, t)=\sin (x$ $+y+t)$, as in [34].

For the convergence of the approximate solution given in (58), we calculate $\alpha_{k}$ by taking any values of $x, y$, and $t$ in the domain of interest. Let us take $x=y=\pi / 8$ and $t=0.25$, we obtain $\alpha_{0}=0.25<1, \alpha_{1}=0.125<1, \alpha_{2}=0.083334<1, \alpha_{3}=$ $0.0625<1, \alpha_{4}=0.05<1, \cdots$, and by induction $\alpha_{k}<1$ for all $k \in N \cup\{0\}$. Therefore, by Definition 8, the solution of Equation (52) converges to the exact solution.
Numerical results corresponding to the two-dimensional nonlinear sine-Gordon equation given in Example 3 are depicted in Table 4 and Figure 3.

Example 4. Consider the following two dimensional sineGordon equation [33]

$$
\frac{\partial^{2} u}{\partial t^{2}}=\frac{\partial^{2} u}{\partial x^{2}}+\frac{\partial^{2} u}{\partial y^{2}}-\sin u,(x, y) \in[-7,7]^{2}, t \geq 0
$$

subject to the initial conditions

$$
\begin{aligned}
u(x, y, 0) & =4 \tan ^{-1}\left(e^{x+y}\right), \\
\frac{\partial}{\partial t} u(x, y, 0) & =-\frac{4 e^{x+y}}{1+e^{2 x+2 y}} .
\end{aligned}
$$

Applying RDTM technique to Equation (59), we obtain the following iterative formula:

$$
(k+2)(k+1) U_{k+2}(x, y)=\frac{\partial^{2}}{\partial x^{2}} U_{k}(x, y)+\frac{\partial^{2}}{\partial y^{2}} U_{k}(x, y)-F_{k}(x, y),
$$

where $F_{k}(x, y)$ is the reduced differential transform of nonlinear term $\sin u(x, y, t)$.

Using RDTM to initial condition (60) and (61), we get

$$
\begin{gathered}
U_{0}(x, y)=4 \tan ^{-1}\left(e^{x+y}\right) \\
U_{1}(x, y)=-\frac{4 e^{x+y}}{1+e^{2 x+2 y}}
\end{gathered}
$$

Applying Lemma 4 and using Equations (63) and (64) in Equation (62), we get the following successive values of $U_{k}($ $x, y)$ fork $(k=0,1,2, \cdots)$.

$$
\left.\begin{array}{l}
U_{2}=2\left[\frac{e^{x+y}-e^{3 x+3 y}}{\left(1+e^{2 x+2 y}\right)^{2}}\right], \\
U_{3}=-\frac{2}{3}\left[\frac{e^{x+y}-6 e^{3 x+3 y}+e^{5 x+5 y}}{\left(1+e^{2 x+2 y}\right)^{3}}\right], \\
U_{4}=\frac{1}{6}\left[\frac{e^{x+y}-23 e^{3 x+3 y}+23 e^{5 x+5 y}-e^{7 x+7 y}}{\left(1+e^{2 x+2 y}\right)^{4}}\right], \\
U_{5}=-\frac{1}{30}\left[\frac{e^{x+y}-76 e^{3 x+3 y}+230 e^{5 x+5 y}-76 e^{7 x+7 y}+e^{9 x+9 y}}{\left(1+e^{2 x+2 y}\right)^{5}}\right], \\
\mathrm{U}_{6}=\frac{1}{180}\left[\left(\frac{e^{x+y}-237 e^{3 x+3 y}+1682 e^{5 x+5 y}-1682 e^{7 x+7 y}+237 e^{9 x+9 y}-e^{11 x+11 y}}{\left(1+e^{2 x+2 y}\right)^{6}}\right)\right],
\end{array}\right\}
$$




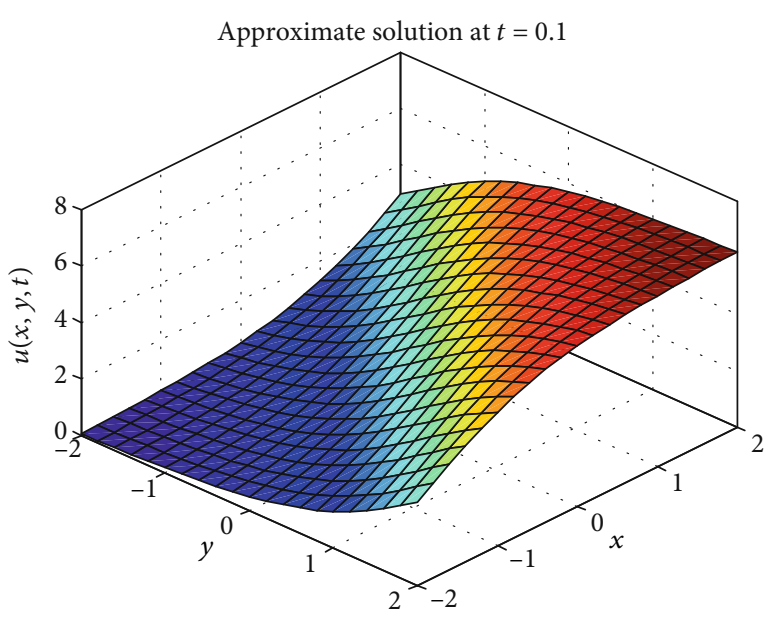

(a)
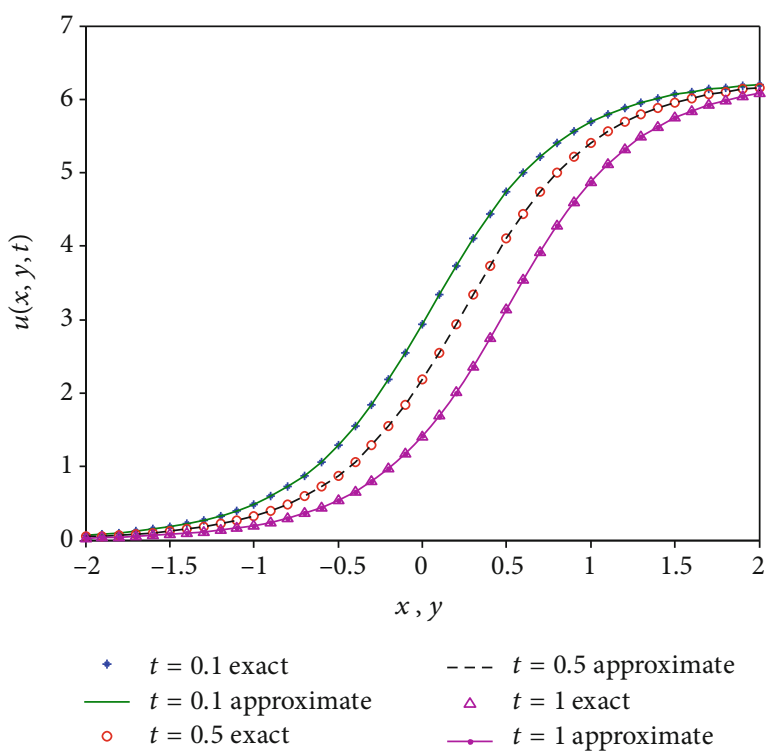

(c)

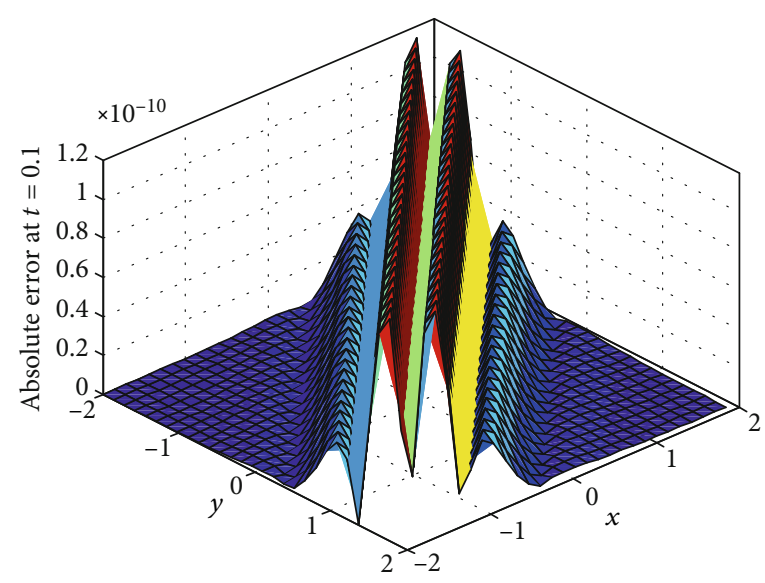

(b)

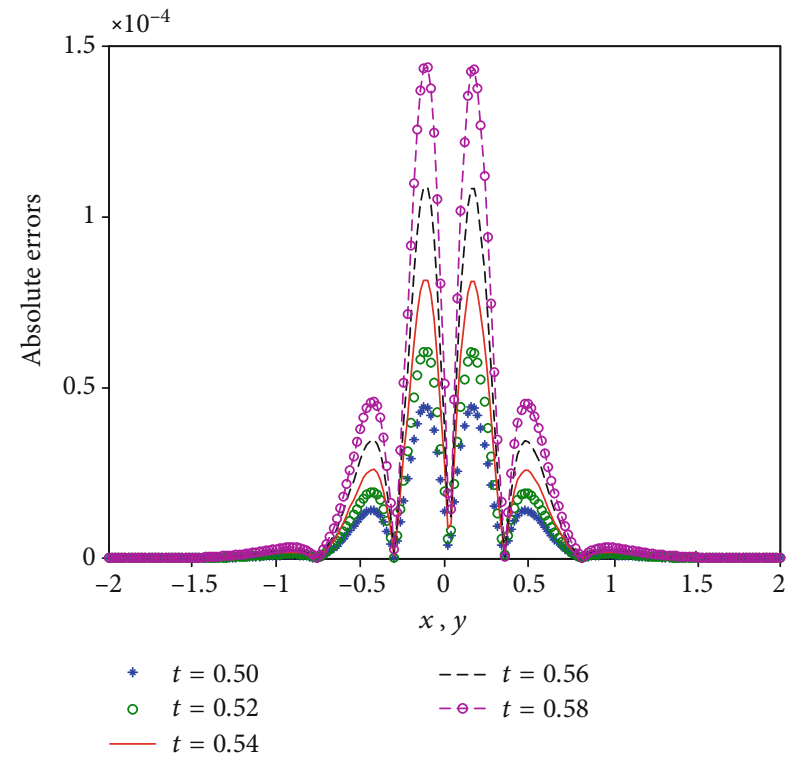

(d)

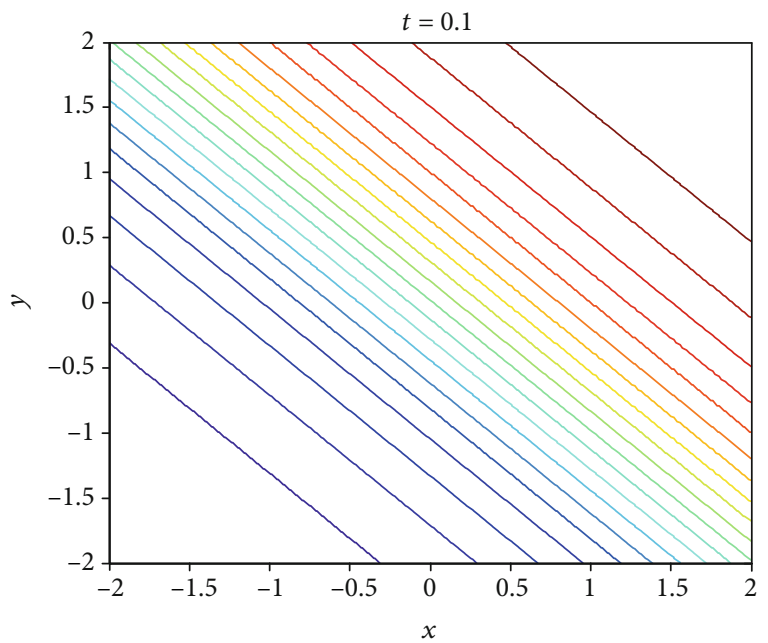

(e)

Figure 4: Plots of the solution behavior of Example 4: (a) approximate solution at $t=0.1$; (b) absolute error $t=0.1$; (c) comparison of exact and approximate solutions for times $t=0.1,0.5,1$; (d) comparison of absolute errors for different values of times $t=0.5,0.52,0.54,0.56,0.58$; and (e) the soliton at $t=0.1$. 
and so on.

Then, the inverse reduced differential transform of $U_{k}($ $x, y)$ gives the $n$-term approximate analytic solution in the form $\tilde{u}_{n}(x, y, t)=\sum_{k=0}^{n} U_{k}(x, y) t^{k}$.

Hence, the exact solution is $u(x, y, t)=\lim _{n \rightarrow \infty} \tilde{u}_{n}(x, y, t)=$ $4 \tan ^{-1}\left(e^{x+y-t}\right)$ as in Shukla et al. [33] and Baccouch [34].

To check the convergence of the approximate analytic solutions, we calculate $\alpha_{k}$ for values $x=y=t=1$, and we obtain $\alpha_{0}=0.00161498087<1, \alpha_{1}=0.48201379002<1, \alpha_{2}$ $=0.29691347980<1, \quad \alpha_{3}=0.16168993395<1 \quad \ldots$ and by induction $\alpha_{k}<1$ for all $k \in N \cup\{0\}$. Therefore, by Definition 8, the solution of Example 4 converges to the exact solution.

Numerical results corresponding to the two-dimensional nonlinear sine-Gordon equation given in Example 4 are depicted in Table 5 and Figure 4.

Tables 2-5 illustrate the approximate analytical solutions of Examples 1-4 obtained by RDTM and the corresponding absolute errors for different values of time $t$. It can be observed from Tables 2-5 that for smaller values of $t$ the corresponding absolute errors are small compared to others. This is to mean that better approximation can be achieved for small values of time $t$ whatever the values of $x$ and $y$ are within the domain of interest.

\section{Graphical Representation and Physical Interpretations}

A graph is a crucial tool to depict the physical structures of the phenomena in the sense of real-world applications. In this section, we have discussed about the obtained solution of the simplified two dimensional sine-Gordon equation using the RDTM method, and we get the travelling wave solutions assembled from Examples 1-4 to the simplified equation. The solutions of examples (1)-(3) and Example 4 are general solitary wave solutions which are periodic wave solution and singular kink shape soliton, respectively. From the above solutions, it has been noted that the solutions (41), (51), and (58) provides periodic wave solution where the solution (65) gives singular kink shape wave solution. Periodic traveling waves play an important role in numerous physical phenomena, including reaction-diffusion-advection systems, self-reinforcing systems, and impulsive systems. Mathematical modeling of many intricate physical events, for instance, physics, mathematical physics, engineering, and many more phenomena resemble periodic traveling wave solutions [46].

Furthermore, Figures 1-4 depict surface plots that show the physical behavior of the RDTM solutions $u(x, y, t)$ and absolute errors of Examples 1-4 for different values of time $t$ in the domain of interest. Specially, in Figures 1(c), 2(c), $3(\mathrm{c})$, and 4(c), comparisons of exact and approximated analytical solutions are compared for different values of times $t$ $=0.1,0.5,1$. As it can be seen from the figures that all the graphs of the approximated analytical solutions for the assigned values of time $t$ resembles to their corresponding graphs of the exact solutions. The comparison of absolute error graphs shown in Figures 1(d), 2(d), 3(d), and 4(d) for different values of times $t=0.50,0.52,0.54,0.56,0.58$ and also the results assert that better approximation for $u(x, y, t$ ) can be obtained when time $t$ is small for any values of $x$ and $y$ in the domain of interest.

\section{Conclusions}

The reduced differential transform method (RDTM) is successfully implemented to find approximate analytical solutions or exact solutions of the two-dimensional nonlinear sine-Gordon equations subject to the appropriate initial conditions. The convergence analysis of the proposed method is also studied, and the results we obtained in Examples 1, 2, 3, and 4 are in excellent agreement with the exact solutions obtained by different methods available in the literature, see Refs. [33, 34, 36, 40, 45]. Furthermore, RDTM is much easier, more convenient, and efficient and this work illustrates the validity and great potential of the reduced differential transform method for solving nonlinear partial differential equations. As a result, the basic ideas of this approach are expected to be further employed to solve other nonlinear problems arising in sciences and engineering.

\section{Data Availability}

The data used to support the findings of this study are available from the corresponding author upon request.

\section{Conflicts of Interest}

The authors declare that there is no conflict of interests about the publication of this paper.

\section{Authors' Contributions}

ATD proposed the main idea of this paper. YOM and AKG supervise his work from the first draft to revision, and approval of the final manuscript for submission.

\section{Acknowledgments}

The authors would like to thank Jimma University, College of Natural Sciences for the supports made.

\section{References}

[1] H. M. Srivastava, V. P. Dubey, R. Kumar, J. Singh, D. Kumar, and D. Baleanu, "An efficient computational approach for a fractional-order biological population model with carrying capacity," Chaos, Solitons and Fractals, vol. 138, article 109880, 2020.

[2] P. Veeresha, D. G. Prakasha, D. Kumar, D. Baleanu, and J. Singh, "An efficient computational technique for fractional model of generalized Hirota-Satsuma-coupled Korteweg-de Vries and coupled Modified Korteweg-de Vries equations," Journal of Computational and Nonlinear Dynamics, vol. 15, no. 7, article 071003, 2020.

[3] F. Ali, Z. Ahmad, M. Arif, I. Khan, and K. S. Nisar, "A time fractional model of generalized Couette flow of couple stress nanofluid with heat and mass transfer: applications in engine oil," IEEE Access, vol. 8, pp. 146944-146966, 2020. 
[4] F. Ali, M. Iftikhar, I. Khan, N. A. Sheikh, and K. S. Nisar, "Time fractional analysis of electro-osmotic flow of Walters's-B fluid with time-dependent temperature and concentration," Alexandria Engineering Journal, vol. 59, no. 1, pp. 25-38, 2019.

[5] F. Ali, N. Khan, A. Imtiaz, I. Khan, and N. A. Sheikh, “The impact of magneto hydrodynamics and heat transfer on the unsteady flow of Casson fluid in an oscillating cylinder via integral transform: a Caputo-Fabrizio fractional model," Paramana, vol. 93, no. 3, 2019.

[6] J. Singh, "Analysis of fractional blood alcohol model with composite fractional derivative," Chaos, Solitons and Fractals, vol. 140, p. 110127, 2020.

[7] J. Singh, D. Kumar, and S. Kumar, “An efficient computational method for local fractional transport equation occurring in fractal porous media," Computational and Applied Mathematics, vol. 39, no. 3, p. 137, 2020.

[8] V. P. Dubey, R. Kumar, D. Kumar, I. Khan, and J. Singh, “An efficient computational scheme for nonlinear time fractional systems of partial differential equations arising in physical sciences," Advances in Difference Equations, vol. 2020, no. 1, 2020.

[9] J. Singh, A. Kilicman, D. Kumar, R. Swroop, and F. M. Ali, "Numerical study for fractional model of nonlinear predatorprey biological population dynamical system," Thermal Science, vol. 23, no. 6, pp. S2017-S2025, 2019.

[10] P. Guo, A. Boldbaatar, D. Yi, and P. Dai, "Numerical solution of sine-Gordon equation with the local kriging meshless method," Mathematical Problems in Engineering, vol. 2020, Article ID 9057387, 11 pages, 2020.

[11] L. De Su, "Numerical solution of two-dimensional nonlinear sine-Gordon equation using localized method of approximate particular solutions," Engineering Analysis with Boundary Elements, vol. 108, pp. 95-107, 2019.

[12] A. Barone, F. Esposito, C. J. Magee, and A. C. Scott, "Theory and applications of the sine-Gordon equation," La Rivista delNuovo Cimento, vol. 1, no. 2, pp. 227-267, 1971.

[13] A. M. Wazwaz, Partial Differential Equations and Solitary Waves Theory, Springer-Verlag, Heidelberg, 2009.

[14] R. J. Cheng and K. M. Liew, "Analyzing two-dimensional sineGordon equation with the mesh-free reproducing kernel particle Ritz method," Computer Methods in Applied Mechanics and Engineering, vol. 245-246, pp. 132-143, 2012.

[15] S. Maitama and Y. F. Hamza, "An analytical method for solving nonlinear sine-Gordon equation," Sohag Journal of Mathematics, vol. 7, no. 1, pp. 5-10, 2020.

[16] M. Dehghan and A. Shokri, "A numerical method for solution of the two-dimensional sine-Gordon equation using the radial basis functions," Mathematics and computers in simulations, vol. 79, pp. 700-715, 2019.

[17] M. Dehghan, M. Abbaszadeh, and A. Mohebbi, "An implicit RBF meshless approach for solving the time fractional nonlinear sine-Gordon and Klein-Gordon equations," Engineering Analysis with Boundary Elements, vol. 50, pp. 412-434, 2015.

[18] M. Dehghan and A. Ghesmati, "Numerical simulation of twodimensional sine-Gordon solitons via a local weak meshless technique based on the radial point interpolation method (RPIM)," Computer Physics Communications, vol. 181, no. 4, pp. 772-786, 2010.

[19] S. M. El-Sayed, "The decomposition method for studying the Klein-Gordon equation," Chaos Solitons \& Fractals, vol. 18, no. 5, pp. 1025-1030, 2003.
[20] D. Kaya, "A numerical solution of the sine-Gordon equation using the modified decomposition method," Applied Mathematics and Computation, vol. 143, no. 2-3, pp. 309-317, 2003.

[21] D. Kaya, "An application of the modified decomposition method for two dimensional sine-Gordon equation," Applied Mathematics and Computation, vol. 159, no. 1, pp. 1-9, 2004.

[22] S. S. Ray, "A numerical solution of the coupled sine-Gordon equation using the modified decomposition method," Applied Mathematics and Computation, vol. 175, no. 2, pp. 1046-1054, 2006.

[23] Q. Wang, "An application of the modified Adomian decomposition method for $(\mathrm{N}+1)$-dimensional sine-Gordon field," Applied Mathematics and Computation, vol. 181, no. 1, pp. 147-152, 2006.

[24] A. Bekir and A. Boz, "Exact solutions for a class of nonlinear partial differential equations using exp-function method," International Journal of Nonlinear Sciences and Numerical Simulation, vol. 8, pp. 505-512, 2007.

[25] A. Aslanov, "The homotopy-perturbation method for solving Klein-Gordon-type equations with unbounded right- hand side," Zeitschrift Fur Naturforschung Section A-A Journal of Physical Sciences, vol. 64, no. 1-2, pp. 149-152, 2009.

[26] M. S. H. Chowdhury and I. Hashim, "Application of homotopy-perturbation method to Klein-Gordon and sineGordon equations," Chaos Solitons \& Fractals, vol. 39, no. 4, pp. 1928-1935, 2009.

[27] D. D. Ganji, A. Sadighi, and B. Ganjavi, "Traveling wave solutions of the sine-Gordon and the coupled sine-Gordon equations using the homotopy-perturbation method," Scientia Iranica Transaction BMechanical Engineering, vol. 16, pp. 189-195, 2009.

[28] U. Yucel, "Homotopy analysis method for the sine-Gordon equation with initial conditions," Applied Mathematics and Computation, vol. 203, no. 1, pp. 387-395, 2008.

[29] A. M. Wazwaz, "The tanh method and a variable separated ODE method for solving double sine-Gordon equation," Physics Letters A, vol. 350, pp. 367-370, 2005.

[30] N. H. Kuo and C. D. Hu, "A study of the solutions of the combined sine-cosine-Gordon equation," Applied Mathematics and Computation, vol. 215, no. 3, pp. 1015-1019, 2009.

[31] B. Batiha, M. S. M. Noorani, and I. Hashim, "Approximate analytical solution of the coupled sine-Gordon equation using the variational iteration method," Physica Scripta, vol. 76, no. 5, pp. 445-448, 2007.

[32] B. Batiha, M. S. M. Noorani, and I. Hashim, "Numerical solution of sine-Gordon equation by variational iteration method," Physics Letters A, vol. 370, no. 5-6, pp. 437-440, 2007.

[33] H. S. Shukla, M. Tamsir, and V. K. Srivastava, "Numerical simulation of two dimensional sine-Gordon solitons using modified cubic B-spline differential quadrature method," AIP Advances, vol. 5, article 017121, 2015.

[34] M. Baccouch, "Optimal error estimates of the local discontinuous Galerkin method the two dimensional Sine-Gordon equation on Cartesian grids," International Journal of Numerical analysis and modeling, vol. 16, no. 3, pp. 436-462, 2019.

[35] Y. Duan, L. Kong, X. Chen, and M. Guo, "Lattice Boltzmann model for two dimensional generalized sine-Gordon equation," Journal of Applied Analysis and Computation, vol. 8, no. 6, pp. 1645-1663, 2018.

[36] D. Li, H. Lai, and B. Shi, "Mesoscopic simulation of the (2+1)dimensional wave equation with nonlinear damping and 
source terms using lattice Boltzmann BGK model," Entropy, vol. 21, no. 390, 2019.

[37] W. G. Belayeh, Y. O. Mussa, and A. K. Gizaw, “Approximate analytic solutions of two-dimensional nonlinear Klein-Gordon equation by using the reduced differential transform method," Mathematical Problems in Engineering, vol. 2020, Article ID 5753974, 12 pages, 2020.

[38] M. Gubes and G. Oturanc, "Approximate solutions of coupled Ramani equation by using RDTM with compared DTM and exact solutions," New Trends in Mathematical Science, vol. 4, no. 4, article 198, 2016.

[39] B. Ibis, "Application of reduced differential transformation method for solving fourth-order parabolic partial differential equations," Journal of Mathematics and Computer Science, vol. 12, no. 2, pp. 124-131, 2014.

[40] X. Kang, W. Feng, K. Cheng, and C. Guo, "An efficient finite difference scheme for the 2D sine-Gordon equation," Journal of nonlinear sciences and applications, vol. 10, no. 6, pp. 2998-3012, 2017.

[41] A. S. Al-Saif and A. J. Harfash, "A comparison between the reduced differential transform method and perturbationiteration algorithm for solving two-dimensional unsteady incompressible Navier-Stokes equations," Journal of Applied Mathematics and Physics, vol. 6, no. 12, pp. 2518-2543, 2018.

[42] M. S. Mohamed and K. A. Gepreel, "Reduced differential transform method for nonlinear integral member of Kadomtsev-Petviashvili hierarchy differential equations," Journal of the Egyptian Mathematical society, vol. 25, no. 1, pp. 1-7, 2017.

[43] K. V. Srivastava, K. M. Awasthi, and K. R. Chaurasia, "Reduced differential transform method to solve two and three dimensional second order hyperbolic telegraph equations," Journal of king Saud university- Engineering sciences, vol. 29, no. 2, pp. 166-171, 2017.

[44] C. B. Neog, "Solution of some system of non-linear partial differential equation using reduced differential transform method," IOSR Journal of Mathematics, vol. 11, no. 5, pp. 37-44, 2015.

[45] W. Liu, J. Sun, and B. Wu, "Space-time spectral method for the two-dimensional generalized sine-Gordon equation," Journal of Mathematical Analysis and applications, vol. 427, no. 2, pp. 787-804, 2015.

[46] A. K. Hossain and M. A. Akbar, "Solitary wave solutions of few nonlinear evolution equations," AIMS Mathematics, vol. 5, no. 2, pp. 1199-1215, 2020. 